\title{
Single incision laparo-endoscopic surgery (SILS) is comparable with robotic surgery at a tertiary care center for the management of gynecologic oncology patients
}

\author{
Evan Cohen, DO ${ }^{1}$ Michael J. Goodheart, MD, ${ }^{1,2}$ David P. Bender, MD ${ }^{1,2}$ Jesus \\ Gonzalez Bosquet MD, PhD, ${ }^{1,2}$ Emily K. Hill, MD ${ }^{1,2}$ Jean-Marie Stephan, MD $^{1,2}$
}

Keywords: Single incision laparoscopy, SILS, robotic surgery, minimally invasive surgery, endometrial cancer

\section{Objectives}

A shift toward minimally invasive surgical techniques has been implemented in the surgical management of gynecologic oncology patients. Over the course of 18 months, we have established a single incision laparo-endoscopic surgery program (SILS), and incorporated it in the management of our patients. We sought to assess the operative and postoperative outcomes of these patients in relation to patients who underwent robotic surgery during that same time period at our institution.

\section{Methods}

We utilized a retrospective chart review to identify 101 patients who since the establishment of our SILS program underwent either robotic surgery or SILS for the management of endometrial cancer. Statistical analyses and plots were generated using $\mathrm{SAS} \otimes$ software. Independent samples t-tests, fisher's exact tests, and simple linear regression were used to compare patient characteristics, operating times, operative complications, blood loss, conversion rates, and pathological findings across the two groups.

${ }^{1}$ Department of Obstetrics and Gynecology, Carver College of Medicine, University of lowa Hospitals and Clinics, lowa City, IA, 52242

${ }^{2}$ Holden Comprehensive Cancer Center, University of lowa Hospitals and Clinics, lowa City, IA, 52242

Please cite this abstract as: Cohen E, Goodheart M, Bender D, Gonzalez Bosquet J, Hill E, Stephan J. Single incision laparo-endoscopic surgery (SILS) is comparable with robotic surgery at a tertiary care center for the management of gynecologic oncology patients. Proc Obstet Gynecol. 2018;8(1):Article 8 [ 2 p.]. Available from: http://ir.uiowa.edu/pog/ Free full text article.

Corresponding author: Evan Cohen, Department of Obstetrics and Gynecology, University of lowa, 200 Hawkins Drive, Iowa City, IA 42242, evan-cohen@uiowa.edu

Copyright: (c) 2018 Cohen, et al. This is an open-access article distributed under the terms of the Creative Commons Attribution License, which permits unrestricted use, distribution, and reproduction in any medium, provided the original author and source are credited. 


\section{Results}

101 patients were included. Sixty-seven patients underwent robotic surgery $(66.3 \%)$, while thirty-four patients underwent SILS (33.7\%). SILS patients had a significantly lower BMI, grade of disease, stage, and fewer co-morbidities than those of the robot group. There were no significant differences in length of hospital stay, blood loss, operative complication rates, conversion rates, or yield of pelvic and para-aortic lymph nodes when comparing the two groups. Operative time was significantly decreased in the SILS group, while both SILS and robotic surgery appeared to have a similar learning curve with consistent improvement in operative time across the study period.

\section{Conclusions}

During the introductory phase of establishing SILS in the treatment of patients with endometrial cancer at our institution, SILS has statistically significant shorter operative time than robotic surgery, and similar length of hospital stay, blood loss, operative and post-operative complications, conversion rates, and yield of lymph node counts. Patients in the SILS groups had a lower BMI and lower grade of their endometrial cancer indicating that with adequate patient selection, this technique could be successfully incorporated for the management of patients with endometrial cancer at a tertiary care center.

Presented at "Advances in Gynecologic Cancers," the University of lowa Obstetrics and Gynecology Postgraduate conference, 10 November 2017, Marriott Coralville Hotel \& Conference Center, Coralville, lowa. 